\title{
Forecast UTI: aplicativo para previsão de leitos de Unidades de Terapia Intensiva no contexto da pandemia de COVID-19*
}

\section{Forecast UTI: application for predicting Intensive Therapy Unit beds in the context of the COVID-19 pandemic}

\section{Forecast UTI: aplicación para prever camas en Unidades de Cuidados Intensivos en el contexto de la pandemia de COVID-19}

\author{
Luiz Leduíno de Salles Neto'- @ orcid.org/0000-0001-8938-5370 \\ Camila Bertini Martins² - (1) orcid.org/0000-0002-8252-8815 \\ Antônio Augusto Chaves'- (1) orcid.org/0000-0001-5767-6798 \\ Thais Cláudia Roma de Oliveira Konstantyner²- @ orcid.org/0000-0002-2289-1866 \\ Horacio Hideki Yanasse'- D orcid.org/0000-0002-6946-9670 \\ Claudia Barbosa Ladeira de Campos'- (1) orcid.org/0000-0002-1836-0915 \\ Ana Júlia de Oliveira Bellini'- (D) orcid.org/0000-0002-1460-4169 \\ Renan Brito Butkeraites'- (D orcid.org/0000-0002-5830-0466 \\ Leonardo Correia'- (1) orcid.org/0000-0002-7091-0435 \\ Igor Luciano Magro'- (1) orcid.org/0000-0003-4671-5290 \\ Fernando dos Santos Soares'- (1) orcid.org/0000-0002-2773-2696 \\ 'Universidade Federal de São Paulo, Instituto de Ciência e Tecnologia, São José dos Campos, SP, Brasil \\ ¿Universidade Federal de São Paulo, Escola Paulista de Medicina, Departamento de Medicina Preventiva, São Paulo, SP, Brasil
}

\section{Resumo}

Frente à necessidade de gerenciamento e previsão do número de leitos de Unidades de Terapia Intensiva (UTI) para pacientes graves de COVID-19, foi desenvolvido o Forecast UTI, um aplicativo de livre acesso, que permite o monitoramento de indicadores hospitalares com base em dados históricos do serviço de saúde e na dinâmica temporal dessa epidemia por coronavírus. 0 Forecast UTI também possibilita realizar previsões de curto prazo do número de leitos ocupados pela doença diariamente, e estabelecer possíveis cenários de atendimento. Este artigo apresenta as funções, modo de acesso e exemplos de uso do Forecast UTI, uma ferramenta computacional destinada a auxiliar gestores de hospitais da rede pública e privada do Sistema Único de Saúde no subsídio à tomada de decisão, de forma rápida, estratégica e eficiente.

Palavras-chave: Infecções por Coronavirus; Vigilância em Saúde Pública; Previsões; Sistema Único de Saúde; Unidades de Terapia Intensiva.

*O projeto de estudo'Estimativa do número de leitos de UTI necessário por dia e gestão de equipamentos durante a epidemia de Covid-19 em diversos cenários' foi aprovado pela Comissão Nacional de Ética em Pesquisa da Universidade Federal de São Paulo e Hospital São Paulo (CEP/UNIFESP e HSP): Protocolo no 6403040420.

\section{Endereço para correspondência:}

Camila Bertini Martins - Universidade Federal de São Paulo, Escola Paulista de Medicina, Departamento de Medicina Preventiva, Rua Botucatu, $n^{\circ} 740,4^{\circ}$ andar, São Paulo, SP, Brasil. CEP: 04023-062

E-mail: cb.martins@unifesp.br 


\section{Introdução}

Desde o início da epidemia pelo COVID-19 em Wuhan, capital da província de Hubei na China, em dezembro de 2019, até 30 de junho de 2020, a doença infectou mais de 10 milhões de pessoas e causou, aproximadamente, 500 mil óbitos em todo o mundo. ${ }^{1}$ No Brasil, segundo dados oficiais, até o dia 20 de junho de 2020, foram registrados mais de 1 milhão de casos e 50 mil óbitos. ${ }^{2}$

Sabe-se que a COVID-19 é uma doença de alta transmissibilidade, sua prevenção e controle estão restritos às medidas não farmacológicas e não existe medicamento comprovadamente eficaz para curar a doença; vacinas para sua prevenção ainda se encontram em fase de estudo. Além da infecção assintomática, o vírus SARS-CoV-2, agente etiológico da doença, é capaz de provocar síndrome respiratória aguda, variando desde casos leves (cerca de 80\%) a casos muito graves (entre 5 e 10\%), que desenvolvem insuficiência respiratória e requerem assistência médica hospitalar. A letalidade da COVID-19, definida como a proporção de casos que evoluíram a óbito, varia conforme a faixa etária e comorbidades do indivíduo infectado, e é influenciada pela capacidade de atendimento do serviço de saúde. ${ }^{3}$

Nesse contexto, umas das principais indagações de gestores de hospitais do Sistema Único de Saúde (SUS) do Brasil é prever o número de leitos de unidade de terapia intensiva (UTI) necessário para atender os casos graves da doença, considerando a dinâmica temporal da epidemia. Com o propósito de auxiliar a tomada de decisão em Saúde Pública, pesquisadores têm desenvolvido ferramentas computacionais ${ }^{4,5}$ que, mediante aplicação de modelos matemáticos, são capazes de estimar o número diário de novos casos de COVID-19, com base na distribuição e gravidade da doença, de modo a prever seu impacto na capacidade de oferta de leitos de UTI. Resumidamente, tais metodologias fazem previsões de curto prazo com base em dados oficiais de saúde, e por meio de aplicativos online, permitem aos usuários ajustarem esses dados conforme as necessidades locais. ${ }^{6,7}$

Considerando-se a distribuição desigual de leitos hospitalares do SUS no território nacional e o fato de a epidemia de COVID-19 encontrar-se em diferentes fases, segundo os municípios e os estados brasileiros, o gerenciamento de leitos de UTI é um desafio a ser enfrentado conforme as especificidades e realidades de um território. ${ }^{8}$

Este artigo apresenta as funções, modo de acesso e uso do aplicativo Forecast UTI, uma ferramenta computacional capaz de auxiliar gestores de hospitais da rede pública e privada do SUS a prever a quantidade de leitos de UTI necessários para 0 atendimento dos casos graves da doença em um dado horizonte de tempo, com base em dados históricos do serviço de saúde.

\section{O aplicativo Forecast UTI}

\section{Desenvolvimento do software}

0 software foi desenvolvido nas seguintes linguagens de programação:

a) SQL para o desenvolvimento do banco de dados

b) Angular Framework para o desenvolvimento da interface com o usuário (front-end)

c) C\# para o desenvolvimento dos algoritmos (back-end)

Em ciência da computação, os termos front-end $\mathrm{e}$ back-end referem-se às etapas inicial e final do processo, respectivamente.

\section{Estatísticas e previsões feitas pelo Forecast UTI}

0 Forecast UTI calcula a média e o desvio-padrão para as variáveis 'número de hospitalização por dia' e 'tempo de internação' (em dias). Para a variável 'letalidade hospitalar', definida como a proporção de casos de COVID-19 que evoluíram a óbito, é apresentado o percentual. 0 aplicativo apresenta a visualização gráfica das distribuições das variáveis 'número de leitos de UTI ocupados por dia', 'número de novas internações por dia', 'número de internações acumulado' e 'número de óbitos por faixa etária', por meio de gráficos de barras. Para as distribuições das variáveis 'idade dos pacientes' (em anos) e 'tempo de internação', são apresentados gráficos de tipo boxplot. Por fim, a variável 'situação atual dos pacientes', classificada como 'internado', 'alta' ou 'óbito', é visualizada da por um gráfico de rosca.

0 Forecast UTI utiliza modelos de regressão polinomial ${ }^{9}$ ajustados até quinta ordem, na previsão do 'número de leitos de UTI ocupados por COVID-19'. Para cada modelo, 0 aplicativo calcula a raiz do erro quadrado médio de previsão (RMSE) e escolhe o modelo com menor RMSE. Nas análises estatísticas, é considerado o erro tipo I de 5\%. 


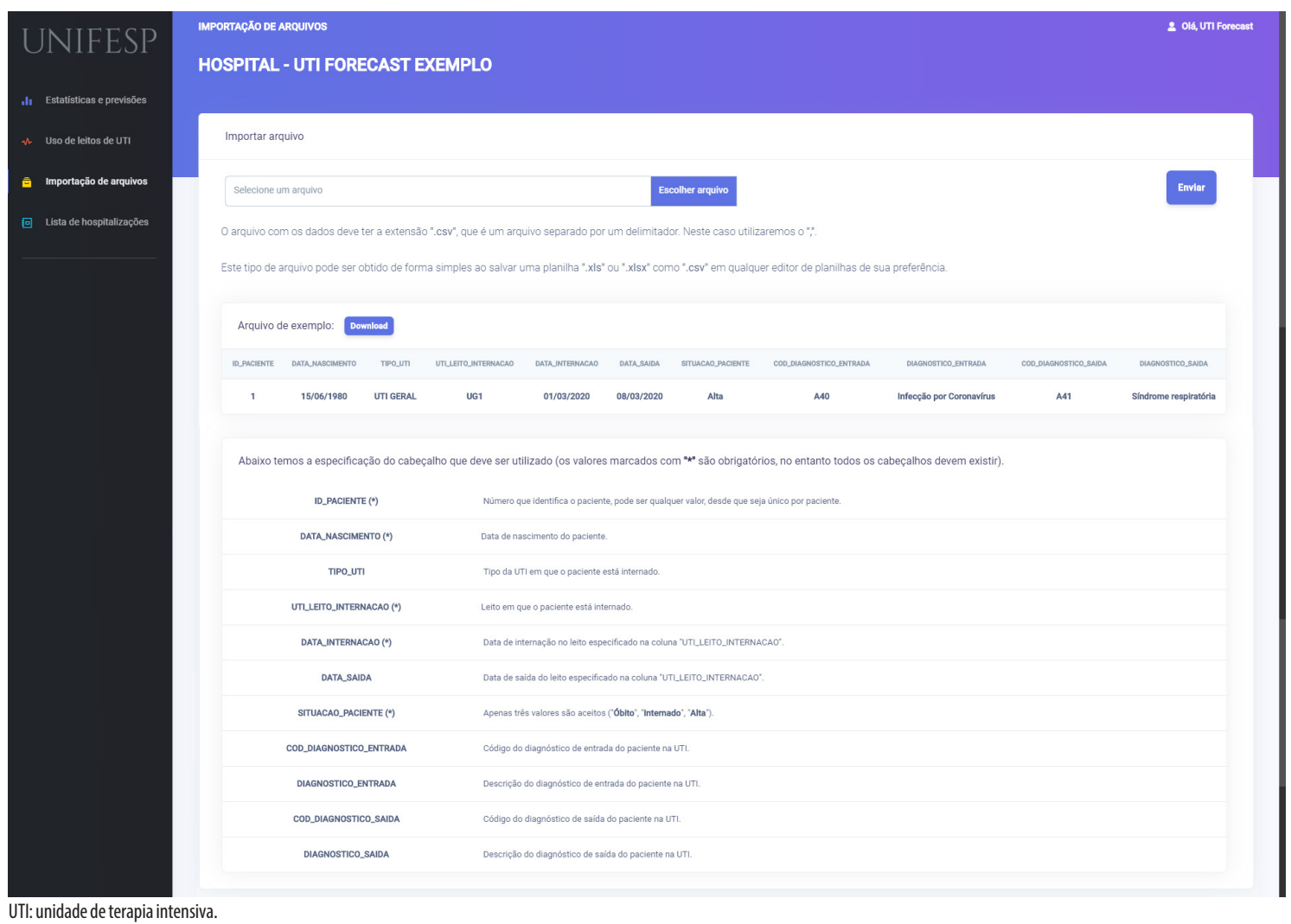

\section{Figura 1 - Aba 'Importação de arquivos' do Forecast UTI: explicação e exemplificação de como montar o banco de dados} e importá-lo

Simulação de cenários realizada pelo Forecast UTI: uso de leitos de UTI

Com base nas variáveis 'número total de leitos', 'número de dias de planejamento', 'média de pacientes por dia', 'tempo de internação' e 'número de leitos ocupados por COVID-19', o aplicativo realiza simulações de cenários hipotéticos de atendimento. Para cada cenário, parâmetros são gerados aleatoriamente, e com base nos dados de entrada fornecidos pelo usuário. 0 número diário de pacientes novos é calculado considerando-se uma distribuição de Poisson, enquanto para o cálculo dos tempos de internação dos novos pacientes, é considerada uma distribuição Uniforme.

Nas simulações, são gerados 1000 cenários diferentes para 0 conjunto de variáveis de entrada. 0 planejamento de cada cenário é executado com o objetivo de alocar o novo paciente no primeiro leito de UTI disponível. Se todos os leitos já estiverem ocupados, o paciente não será atendido pelo hospital. Após simular todos os cenários, a ferramenta apresenta o pior cenário possível, ou seja, aquele que resulta no maior número de pacientes que não podem ser atendidos devido à ocupação máxima dos leitos de UTI.

\section{Acesso ao Forecast UTI}

0 gestor do SUS interessado no aplicativo deve preencher o formulário disponível em https://www. forecastuti.unifesp.br/

Posteriormente ele receberá um nome de usuário e uma senha, a serem utilizados para acesso online gratuito.

\section{Importação de arquivos no Forecast UTI}

Após acessar o aplicativo, na aba 'Importação de arquivos', o usuário deverá carregar o sistema com um arquivo de extensão de tipo '.csv', contendo as variáveis obrigatórias de seus pacientes: 'número de identificação', 'data de nascimento', 'leito de internação', 'data de internação' e 'situação atual do paciente'. Se o usuário desejar, também é possivel inserir dados referentes às variáveis 'tipo da UTI', 'CID de entrada'e 'CID de saída' do paciente (Figura 1). É garantido o sigilo dos dados carregados no website, que permanecem disponíveis exclusivamente para o usuário cadastrado.

\section{Exemplos de uso do Forecast UTI}

A título de ilustração, foram utilizados dados artificiais para exemplificação dos recursos do Forecast UTI. 


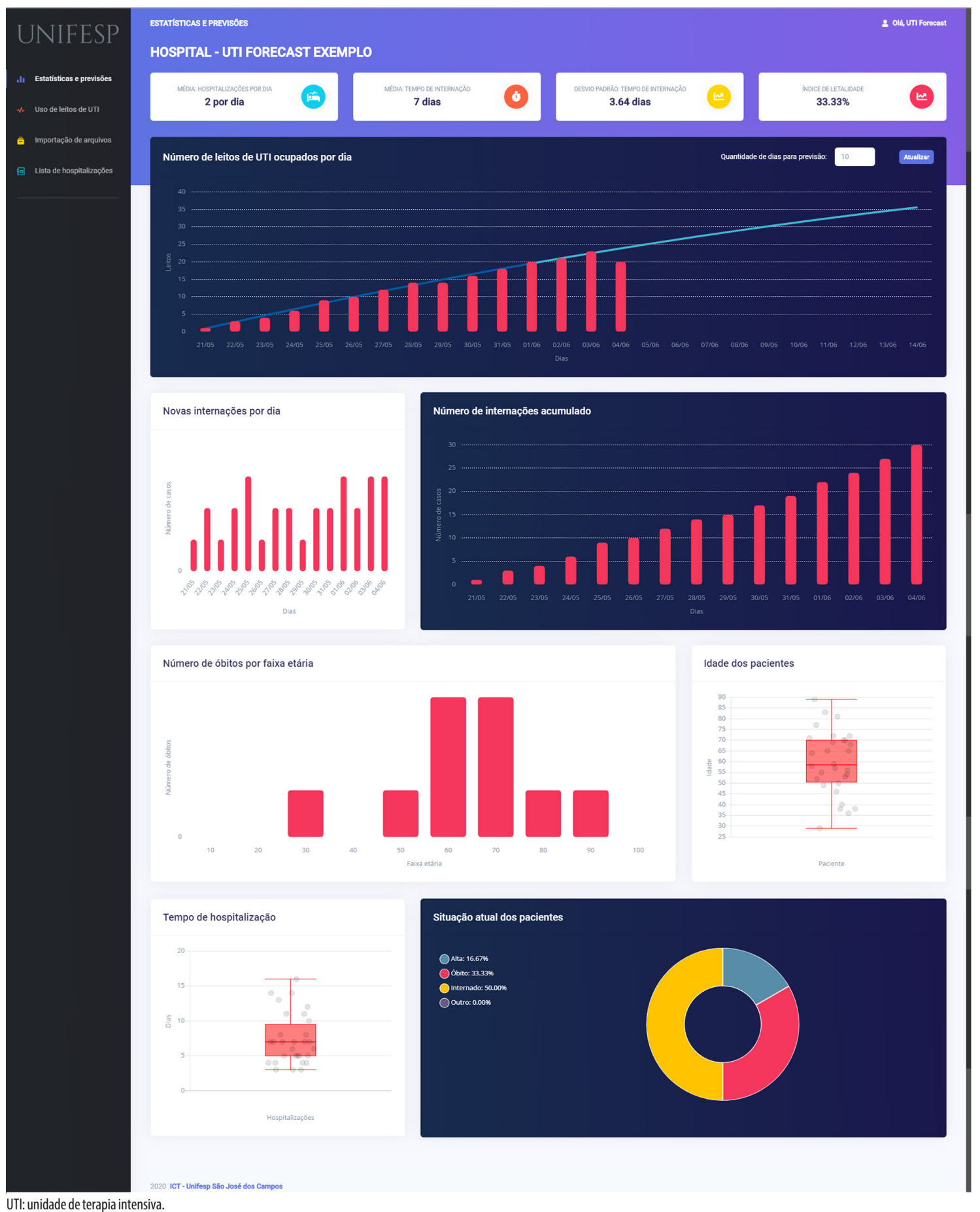

UTI: unidade de terapia intensiv.

Figura 2 - Aba 'Estatísticas e previsões' da ferramenta Forecast UTI, utilizando-se dados artificiais: indicadores hospitalares de interesse e previsão do número de leitos de UTI ocupados por dia 


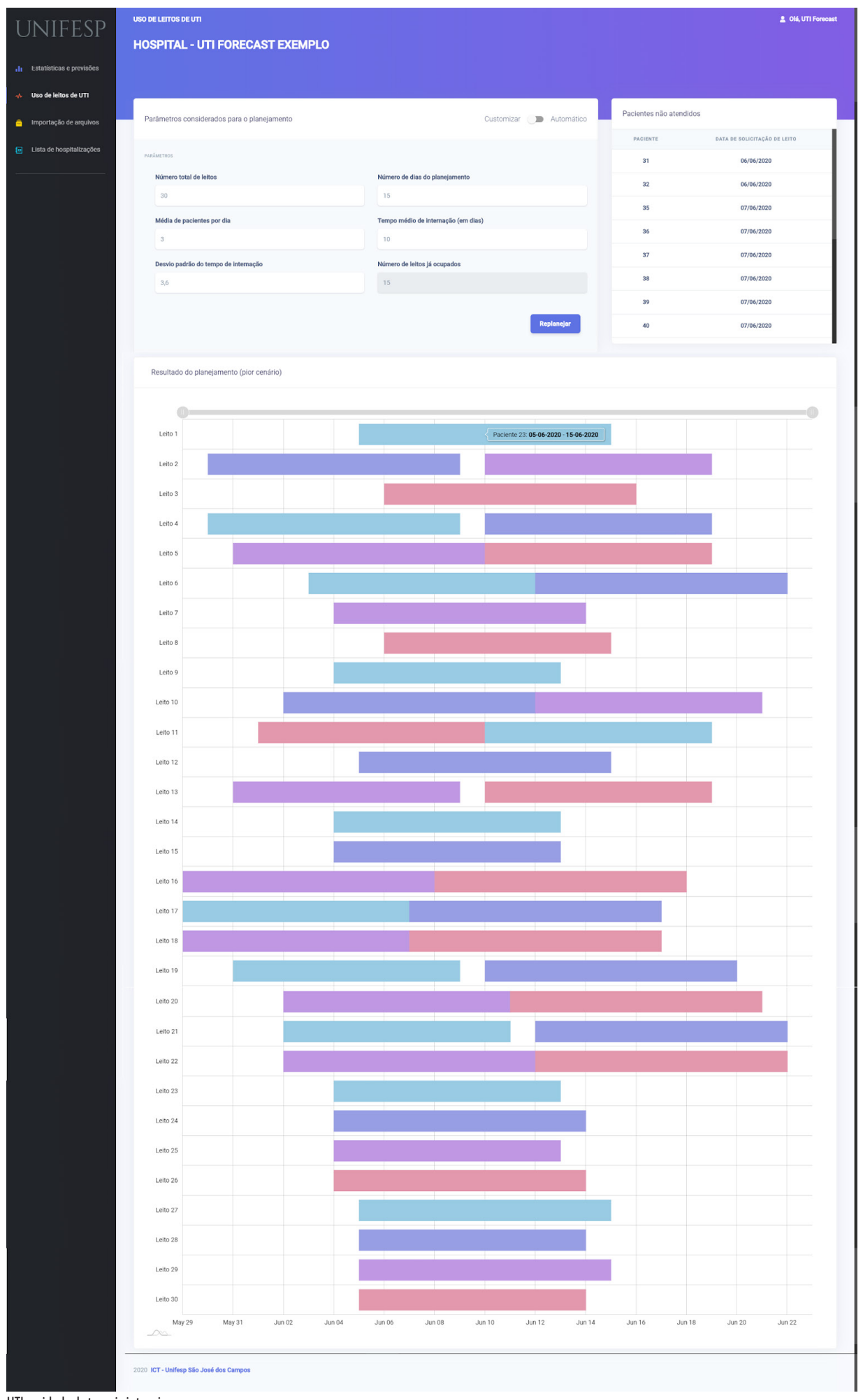

UTI: unidade de terapia intensiva.

Figura 3 - Aba 'Uso de leitos de UTI' da ferramenta Forecast UTI, utilizando dados artificiais: simulação da ocupação dos leitos, considerando parâmetros customizados o maior número de pacientes não atendidos devido à ocupação máxima dos leitos de UTI (pior cenário) 
As informações de login (exemplo@unifesp.br) e senha (dadosexemplo) podem ser utilizadas para acessar o site do aplicativo, caso o leitor queira reproduzir os exemplos a seguir.

A ferramenta disponibiliza duas abas principais de monitoramento: 'Estatística e previsões' e 'Uso de leitos de UTI', que permitem o acompanhamento da ocupação dos leitos de UTI por COVID-19 e a simulação de diferentes cenários de atendimento, respectivamente.

Na aba 'Estatística e previsões', visualiza-se a média diária de internações na UTI, a média do tempo de hospitalização na UTI e a letalidade por COVID-19. Pode-se observar a distribuição do número de leitos de UTI ocupados por diae realizar previsões futuras de até 10 dias. Nessa aba, também é possivel visualizar a distribuição de novas internações por COVID-19, por dia, e do número de internações acumulado, o monitoramento do número de óbitos por faixa etária, a distribuição dos pacientes segundo faixa etária, o tempo de hospitalização e a situação atual (Figura 2).

A aba 'Uso de leitos de UTI' (Figura 3) permite a simulação de diferentes cenários, para auxiliar no gerenciamento dos leitos de UTI. Essa simulação possibilita verificar o que acontecerá com a ocupação dos leitos de UTI se houver mudança no número de novos pacientes por dia, o tempo médio de internação e o número de leitos disponíveis. 0 aplicativo apresenta cada leito de UTI em uma linha do gráfico, além dos pacientes internados, nos respectivos leitos, ao longo do horizonte de planejamento. As barras representam as datas de entrada e saída de cada paciente. Também são apresentados os pacientes que não foram internados por ausência de leitos de UTI disponíveis no dia quando chegaram ao hospital. Na Figura 3, finalmente, é apresentada, graficamente, a situação no pior cenário da simulação, realizada com dados de teste. Neste exemplo, foram utilizados os seguintes valores: número total de leitos = 30 leitos; número de dias do planejamento $=15$ dias; média de pacientes por dia $=3$ pacientes; tempo médio de internação $=10$ dias; desviopadrão do tempo de internação $=3,6$ dias; e número de leitos já ocupados $=15$ leitos. Para essa simulação, 0 pior cenário encontrado resultou em 16 pacientes sem atendimento por falta de leitos.

\section{Considerações finais}

Apresentou-se 0 aplicativo Forecast UTI e suas aplicações para o gerenciamento de leitos de UTI do SUS. Tal ferramenta foi desenvolvida para suprir a necessidade do acompanhamento dos leitos hospitalares durante a pandemia de COVID-19 e facilitar a gestão de hospitais públicos e privados da rede SUS.

A aba 'Estatísticas e previsões' torna possível a visualização e monitoramento de informações de pacientes internados em UTI por COVID-19, auxiliando na gestão de leitos de UTI, no contexto de epidemias. A aba 'Uso de leitos de UTI' possibilita a simulação de diferentes cenários de alocação dos pacientes, resultando o cenário com maior número de pacientes não atendidos por conta da ocupação máxima dos leitos de UTI.

Cabe destacar que a ferramenta Forecast UTI é gratuita, sigilosa e flexível, podendo ser utilizada por qualquer gestor interessado. Uma vez que os dados são inseridos pelo próprio usuário e a plataforma realiza as alterações automaticamente, sempre será possível ter acesso às estatísticas básicas dos pacientes, previsões e simulações em tempo real. 0 uso do aplicativo é intuitivo e amigável, porém melhorias e modernizações sempre são possíveis, como a possibilidade de (i) carregar arquivos com outras extensões, (ii) implementar outros modelos de previsão, tais como modelos polinomiais fracionários e modelos de machine learning, (iii) inserir novas variáveis de interesse, como por exemplo, a presença de comorbidades, além de (iv) permitir a integração do sistema com o prontuário eletrônico do paciente e (v) traduzir sua linguagem para outros idiomas. Finalmente, é importante ressaltar que a precisão das estatísticas e previsões realizadas depende da qualidade dos dados inseridos na plataforma.

No momento da conclusão deste manuscrito, são aproximadamente 20 os usuários do aplicativo Forecast UTI, entre hospitais, secretarias municipais e de Estado de saúde e pesquisadores, e até então, não foram reportados problemas na utilização do software.

Acredita-se que ferramentas como a Forecast UTI são úteis para o gerenciamento de serviços hospitalares da rede pública e privada do Sistema Único de Saúde, uma vez que subsidiam a tomada de decisão de forma rápida, estratégica e eficiente. Espera-se que o presente trabalho possa contribuir para 0 aprimoramento e a expansão do uso de tecnologias dessa natureza.

\section{Contribuição dos autores}

Salles Neto LL, Martins CB, Chaves AA, Konstantyner TCR0, Yanasse HH e Campos CBL foram responsáveis pela concepção e delineamento do estudo, análise e interpretação dos dados, redação e revisão crítica do conteúdo do manuscrito. Bellini AJO, Butkeraites 
RB, Correia L, Magro IL e Soares FS contribuíram na análise e interpretação dos dados, redação e revisão crítica do conteúdo do manuscrito. Todos os

\section{Referências}

1. Health Organization - WHO. Coronavirus disease: situation report - 162 [Internet]. Geneva: World Health Organization; 2020 [cited 2020 Jul 27]. Available from: https://www.who. int/docs/default-source/coronaviruse/20200630-covid-19sitrep-162.pdf?sfvrsn=e00a5466_2

2. Ministério da Saúde (BR). Secretaria de Vigilância em Saúde. Doença pelo coronavírus COVID-19. Bol Epidemiol [Internet]. 2020 jun [citado 2020 jul 27];19(especial). Disponível em: http://saude.gov.br/images/pdf/2020/June/25/ Boletim-epidemiologico-COVID-19-2.pdf

3. Ministério da Saúde (BR). Guia de vigilância epidemiológica: emergência de saúde pública de importância nacional pela doença pelo coronavírus 2019 [Internet]. Brasília: Ministério da Saúde; 2020 [citado 2020 jul 27]. Disponível em: https:// portalarquivos.saude.gov.br/images/pdf/2020/April/07/ GuiaDeVigiEpidemC19-v2.pdf

4. Nedel FB. csapAIH: uma função para a classificação das condições sensíveis à atenção primária no programa estatístico R. Epidemiol Serv Saúde [Internet]. 2017 jan-mar [citado 2020 jul 27];26(1):199-209. Disponível em: http:// dx.doi.org/10.5123/s1679-49742017000100021

5. Saldanha RF, Bastos RR, Barcellos C. Microdatasus: pacote para download e pré-processamento de microdados

\section{Abstract}

In view of the need to manage and forecast the number of Intensive Care Unit (ICU) beds for critically ill COVID-19 patients, the Forecast UTI open access application was developed to enable hospital indicator monitoring based on past health data and the temporal dynamics of the Coronavirus epidemic. Forecast UTI also enables short-term forecasts of the number of beds occupied daily by COVID-19 patients and possible care scenarios to be established. This article presents the functions, mode of access and examples of uses of Forecast UTI, a computational tool intended to assist managers of public and private hospitals within the Brazilian National Health System by supporting quick, strategic and efficient decision-making.

Keywords: Coronavirus Infections; Public Health Surveillance; Forecasting; Unified Health System; Intensive Care Units. autores aprovaram a versão final do manuscrito e são responsáveis por todos seus aspectos, incluindo a garantia de sua precisão e integridade. do Departamento de Informática do SUS (DATASUS). Cad Saúde Pública [Internet]. 2019 [citado 2020 jul 27];35(9):e00032419. Disponível em: http://dx.doi. org/10.1590/0102-311x00032419

6. DeasyJ, Rocheteau E, Kohler K, Stubbs DJ, Barbiero P, Liò $\mathrm{P}$, et al. Forecasting ultra-early intensive care strain from COVID-19 in England. MedRxiv [Internet]. 2020 Apr [cited 2020 Jul 27]. Available from: https://doi.org/10.1101/2020.0 3.19.20039057

7. Giannakeas V, Bathia D, Warkentin MT, Bogosh I, Stall MN. Estimating the maximum daily number of incident COVID-19 cases manageable by a healthcare system. MedRxiv [Internet]. 2020 Mar [cited 2020 Jul 27]. Available from: https://doi.org/10.1101/2020.03.25.20043711

8. Barcellos CC, Sabroza PC, Peiter P, Rojas LI. Organização espacial, saúde e qualidade de vida: análise espacial e uso de indicadores na avaliação de situações de saúde. Inf Epidemiol SUS [Internet]. 2002 set [citado 2020 jul 27];11(3):129-38. Disponível em: https://www.arca.fiocruz.br/handle/icict/713

9. Lindley DV. Regression and correlation analysis. In: Eatwell J, Milgate M, Newman P, editors. Time series and statistics. London: Palgrave Macmillan; 1990

\section{Resumen}

Envista de la necesidad de administrary prever el número de camasen la Unidad de Cuidados Intensivosparapacientes graves de COVID-19, se desarrolló Forecast UTI: una aplicación de acceso abierto que permite el monitoreo de indicadores hospitalarios basados en datos históricos del servicio salud y la dinámica temporal de esta epidemia por coronavirus También es posible hacer pronósticos a corto plazo del número de camas ocupadas diariamentepor la enfermedady establecerposibles escenarios de atención. Este artículo presenta las funciomes, el modo de acceso y ejemplos de uso de Forecast UII, una herramienta computacional capaz de aynudar a los gestores de hospitalespúblicosy privadosen el Sistema Único de Salud, ya que apoyan la toma de decisiones de manera rápida, estratégica y eficiente.

Palabras clave: Infecciones por Coronavirus; Vigilancia en Salud Pública; Predicción; Sistema Único de Salud; Unidades de Cuidados Intensivos.

Recebido em 06/06/2020

Aprovado em 22/07/2020 


\section{Errata}

No artigo "Forecast UTI: aplicativo para previsão de leitos de Unidades de Terapia Intensiva no contexto da pandemia de Covid-19”, com número de doi: 10.5123/s1679-49742020000400023, publicado no periódico Epidemiologia e Serviços de Saúde, 29(4):1-7, na página 1:

Onde se lia:

“doi: 10.5123/s1679-49742020000400023”

Leia-se:

"doi: 10.1590/s1679-49742020000400023" 